\title{
Transcriptome analysis of sinensetin-treated liver cancer cells guided by biological network analysis
}

\author{
SEONG MIN KIM ${ }^{1}$, SHAILIMA RAMPOGU ${ }^{2}$, PREETHI VETRIVEL ${ }^{1}$, APOORVA M. KULKARNI ${ }^{2}$, \\ SANG EUN HA ${ }^{1}$, HUN HWAN KIM ${ }^{1}$, KEUN WOO LEE ${ }^{2}$ and GON SUP KIM ${ }^{1}$ \\ ${ }^{1}$ Research Institute of Life Science and College of Veterinary Medicine; ${ }^{2}$ Division of Life Science, \\ Division of Applied Life Science (BK21 Plus), Plant Molecular Biology and Biotechnology Research Center, \\ Research Institute of Natural Science, Gyeongsang National University, Jinju, Gyeongsangnam 52828, Republic of Korea
}

Received July 28, 2020; Accepted February 15, 2021

DOI: $10.3892 / 01.2021 .12616$

\begin{abstract}
Hepatocellular carcinoma is recognized as one of the most frequently occurring malignant types of liver cancer globally, making the identification of biomarkers critically important. The aim of the present study was to identify the genes involved in the anticancer effects of flavonoid compounds so that they may be used as targets for cancer treatment. Sinensetin (SIN), an isolated polymethoxyflavone monomer compound, possesses broad antitumor activities in vitro. Therefore, the identification of a transcriptome profile on the condition of cells treated with SIN may aid to better understand the genes involved and its mechanism of action. Genomic profiling studies of cancer are increasing rapidly in order to provide gene expression data that can reveal prognostic biomarkers to combat liver cancer. In the present study, high-throughput RNA sequencing (RNA-seq) was performed to reveal differential gene expression patterns between SIN-treated and SIN-untreated human liver cancer HepG2 cells. A total of 43 genes were identified to be differentially expressed (39 downregulated and 4 upregulated in the SIN-treated group compared with the SIN-untreated group). An extensive network analysis for these 43 genes resulted in the identification of 10 upregulated highly interconnected hub genes that contributed to the progression of cancer. Functional enrichment analysis of these 10 hub genes revealed their involvement in the regulation of apoptotic processes, immune response and tumor necrosis factor production. Additionally, the mRNA expression levels of these 10 genes were evaluated using reverse transcription-quantitative PCR, and the results were consistent with the RNA-seq data. Overall, the results of the present study revealed differentially expressed genes
\end{abstract}

Correspondence to: Professor Gon Sup Kim, Research Institute of Life Science and College of Veterinary Medicine, Gyeongsang National University, 501 Jinju-dearo, Jinju, Gyeongsangnam 52828, Republic of Korea

E-mail: gonskim@gnu.ac.kr

Key words: liver cancer, sinensetin, RNA sequencing, transcriptome involved in cancer after SIN treatment in HepG2 cells and may help to develop strategies targeting these genes for treating liver cancer.

\section{Introduction}

Liver cancer is one of the most common cancers with an increasing death rate. Approximately 0.56 million new cases are reported annually (1). Approximately 50\% of patients during treatment with chemotherapy develop metastasis, thus reducing their survival rate (2). Difficulties in chemotherapeutic techniques have led to other treatment options such as the use of nutraceuticals and natural therapies for combating this diseased with prior knowledge about their mechanisms of action (3). Thus, an emerging theme in cancer biology is targeting genes and/or proteins that can link to the progression of cancer and factors that directly or indirectly affect the proliferation and metastasis (4).

Natural herbs have gained attraction in the current era due to their roles in controlling cancer growth by targeting oncogenes and proteins that are altered in cancers. Isolated monomer polymethoxyflavones (PMFs) have shown a broad spectrum of anti-cancer activities by inhibiting cell proliferation and cancer prevention (5). Sinensetin (SIN) is one such polymethoxyflavone present in citrus fruits that has notable anti-inflammatory and anti-tumor activities (6). Although the anti-cancer effect of SIN in cancer cells has been studied recently, gene expression changes and molecular mechanisms associated with its anti-cancer activities remain largely unknown. Transcription profile using RNA-sequencing (RNA-seq) technology can help us understand its mechanism of action (7). Transcriptome analysis is a technology using bioinformatics and data-mining tools to analyze changed target genes and understand the mechanism of action of a drug after treatment with an in vitro model (8). The major way to explore the molecular mechanism involved in the anti-cancer effect of flavonoids is by determining gene activities and functions using transcriptome analysis (9). Genomic findings through a sequencing approach provide features of genomes due to external influences such as a drug treatment. Differential gene expression under treated conditions of a disease can provide us a better understanding of factors in a particular state modified by a candidate drug (10). 
Furthermore, gene expression obtained from transcriptome data can lead to the discovery of novel key genes associated with the related pathway (11). The advent of next-generation sequencing (NGS) data provides a detailed cancer profile that can explain the relationship and connection of genes involved in a disease. In addition, the analysis of differential patterns of genes can help us understand biological processes, cellular components, and interacting pathway network related to cancer pathogenesis for each one (12). Furthermore, bioinformatics analysis of differential gene profiles is an attractive strategy to identify novel therapeutic biomarkers for treating a disease (13). In this regard, taking an integrated approach to identify mRNA targets using next-generation sequencing data can reveal specific biomarkers for cancer types.

Developed almost a decade ago, RNA-seq is a potent tool for understanding genomic functions. Differentially expressed genes (DEG) remains the primary application of RNA-seq (14). DEGs are genes whose expression levels are significantly altered between two or more conditions such as before and after treatment with a drug. In this regard, the concept of hub genes is gaining interest. Hub genes are highly interconnected genes in a protein-protein interaction (PPI) network. Their associated biological process gene ontology terms and pathways might improve our understanding of their roles in carcinogenicity (15).

In the current study, we performed Illumina NovaSeq6000 sequencing for SIN-treated and -untreated HepG2 liver cancer cells and studied differential gene expression patterns. Highly interconnected genes (hub) genes among these were studied extensively for their roles in cancer. The expression of these hub genes was further validated by qRT-PCR.

\section{Materials and methods}

Cell culture. A human liver cancer cell line (HepG2) authenticated using short tandem repeat (STR) profiling was obtained from the Korean Cell Line Bank (Seoul, Korea). HepG2 cells were cultured in DMEM (Gibco; Thermo Fisher Scientific, Inc.) supplemented with $10 \%$ fetal bovine serum (Gibco; Thermo Fisher Scientific, Inc.), $100 \mathrm{U} / \mathrm{ml}$ penicillin, and $100 \mu \mathrm{g} / \mathrm{ml}$ streptomycin (Gibco; Thermo Fisher Scientific, Inc.) at $37^{\circ} \mathrm{C}$ with $5 \% \mathrm{CO}_{2}$.

Isolation of RNA for sequencing. HepG2 cells were seeded into 6-well plates and treated with $100 \mu \mathrm{M}$ of SIN for $48 \mathrm{~h}$ at $37^{\circ} \mathrm{C}$. After $48 \mathrm{~h}$ treatment, total RNAs were extracted using TRIzol. The concentration of RNA was determined using a spectrophotometer. Isolated total RNA was then subjected to sequencing to obtain expression data.

Library preparation and sequencing. Sequencing was performed by TheragenEtex (Gyeonggi-do) with the following described protocol. RNA-seq libraries were constructed using a TruSeq Stranded mRNA Sample Prep Kit (Illumina, Inc.) for $150-b p$ paired-end sequencing. Poly-A-containing mRNA molecules were purified and fragmented from $2 \mu \mathrm{g}$ of DNase-treated total RNA using oligo(dT) magnetic beads. Following the purification step, mRNAs were fragmented and used for synthesis of single-stranded cDNAs by means of random hexamer priming. With the constructed single-stranded cDNAs as templates, second strand cDNA synthesis was carried out to prepare double-stranded cDNAs. These cDNAs were then amplified with a sequential process of end-pair repair, addition of A-tail, and adapter ligation using polymerase chain reaction (PCR). The quality of constructed cDNA libraries wase evaluated with an Agilent 2100 BioAnalyzer (Agilent Technologies, Inc.) and quantified with a KAPA library quantification kit (Kapa Biosystems) according to each manufacturer's protocol. These products were then purified and enriched with cluster amplification using PCR to obtain the final complementary DNA library for high-throughput paired-end (2x150 bp) DNA sequencing using an Illumina NovaSeq6000 (Illumina, Inc.).

Transcript data analysis. After filtering out low quality reads, TopHat was used to map quality-filtered reads to a reference genome (hg38) (16). We measured gene expression levels with Cufflinks v2.1.1 using the Ensembl human gene annotation database. We performed differential expression analysis using cuffdiff (17). To improve the accuracy of measurement, we applied frag bias and multi-read correct options for both cufflinks and cuffdiff. All other options were set to default values. DEGs were identified and filtered with the following criteria: false discovery rate $<0.05$ and $\log _{2} \mathrm{FCl}>1$ (18).

Identification of hub genes and their analysis. To identify hub genes among DEGs, a PPI network of 43 DEGs was constructed using STRING (https://string-db.org/) with a 'minimum required interaction score' set to medium confidence $(0.400)$ and 'maximum number of interactors to show' set to query proteins for both 1st and 2nd shells (19). The PPI network was imported in cytoscape using CytoHubba's Maximal Clique Centrality (MCC) scoring method to identify top ten hub genes incorporated in STRING and to discover significantly enriched biological process gene ontology (GO) terms and KEGG pathways (20). Top ten biological process GO terms with the lowest false discovery rate were analyzed using REVIGO (21). Additionally, we performed an extensive literature survey for these genes to uncover their roles in cancer.

qRT-PCR and data analysis. To validate hub genes differentially expressed based on transcriptome analysis, we studied mRNA expression levels of these hub genes by quantitative real-time polymerase chain reaction (qRT-PCR). HepG2 cells were seeded into 6-well plates and treated with $100 \mu \mathrm{M}$ of SIN for $48 \mathrm{~h}$ at $37^{\circ} \mathrm{C}$. Total RNA was isolated and its concentration was determined using a spectrophotometer. Total RNA $(1 \mu \mathrm{g})$ was converted to cDNA using an iScript ${ }^{\mathrm{TM}}$ cDNA synthesis kit (Bio-Rad Laboratories, Inc.). qRT-PCR was then performed using cDNA and AccuPower ${ }^{\circledR}$ 2X Greenstar ${ }^{\mathrm{TM}}$ qPCR Master (Bioneer). qPCR primers used in this study are listed in Table I. All data were analyzed using Bio-Rad CFX Manager Version 3.1. We measured relative quantification using the $2^{-\triangle \Delta C q}$ method. mRNA expression levels of target genes were normalized against those of $\beta$-actin.

Statistical analysis. All data were analyzed using GraphPad Prism version 5.0 (GraphPad Software). Results are expressed as means \pm SEM. They were evaluated using the Student's t-test. $\mathrm{P}<0.05$ was considered to indicate statistical significance. 
Table I. Forward and reverse primers used for reverse transcription-quantitative PCR of hub genes.

\begin{tabular}{|c|c|}
\hline Gene name & Primer sequence $\left(5^{\prime}-3^{\prime}\right)$ \\
\hline Ceruloplasmin & $\begin{array}{l}\text { F: TGCAAATGGGAGACAGAAAGA } \\
\text { R: TCAGGTGCAGTTGTAAACATTC }\end{array}$ \\
\hline FGB & $\begin{array}{l}\text { F: CAGGACCATGACCATTCACA } \\
\text { R: CATCCACCACCGTCTTCTTTA }\end{array}$ \\
\hline IGF2 & $\begin{array}{l}\text { F: CGGCTTCTACTTCAGCAGG } \\
\text { R: GTAGCACAGTACGTCTCCAG }\end{array}$ \\
\hline ITIH3 & $\begin{array}{l}\text { F: CAGTTGCAGGGCTTCTATGA } \\
\text { R: GCTGGTAAGTGTTCTGGGTG }\end{array}$ \\
\hline NR1H4 & $\begin{array}{l}\text { F: TACCAGGATTTCAGACTTTGGAC } \\
\text { R: CCCAGACGGAAGTTTCTTATTGA }\end{array}$ \\
\hline ORM1 & $\begin{array}{l}\text { F: ACCAGTGCATCTATAACACCAC } \\
\text { R: AGCAAGCATGTAGGTCTTGG }\end{array}$ \\
\hline ORM2 & $\begin{array}{l}\text { F: ACCAGTGCTTCTATAACTCCAG } \\
\text { R: ATCGTCCAGGTAGGAACCAA }\end{array}$ \\
\hline SERPINA7 & $\begin{array}{l}\text { F: ATATGACCTTGGAGCCACAC } \\
\text { R: CCTTATGGGCAGCATTGGAA }\end{array}$ \\
\hline SERPINE1 & $\begin{array}{l}\text { F: GACCCATGACCTGCAGAAAC } \\
\text { R: TACAGGTCCTTCTTGCCTGA }\end{array}$ \\
\hline THBS1 & $\begin{array}{l}\text { F: CCTGGACTCGCTGTAGGTTA } \\
\text { R: CTGGACTGGTAGCCAAAGAC }\end{array}$ \\
\hline$\beta$-actin & $\begin{array}{ll}\text { F: } & \text { TTCTACAATGAGCTGCGTGTGG } \\
\text { R: } & \text { GTGTTGAAGGTCTCAAACATGAT }\end{array}$ \\
\hline
\end{tabular}

F, forward; R, reverse; FGB, fibrinogen $\beta$ chain; IGF2, insulin-like growth factor 2 ; ITIH3, inter- $\alpha$-trypsin inhibitor heavy chain 3; $\mathrm{NRIH} 4$, nuclear receptor subfamily 1 group $\mathrm{H}$ member 4; ORM1/2, orosomucoid 1/2; SERPINE1, serpin family E member 1; SERPINA7, serpin family A member 7; THBS1, thrombospondin 1.

\section{Results}

Identification of DEGs. In our previous study on SIN treatment in HepG2 cells, we found that SIN could induce HCC cell death in vitro (22). RNA-seq and Cuffdiff identified 43 DEGs (Table II) between SIN-untreated and SIN-treated HepG2 cells based on the selection criteria (a false discovery rate $<0.05$ and $\log _{2} \mathrm{FCl}>1$ ). Out of these 43 DEG's, 39 were downregulated and 4 were upregulated. These DEGs were plotted using the heatmap.2 function from R's gplot package. Fragments per kilobase of transcript per million (FPKM) values per genes in SIN-untreated (control) and SIN-treated (case) HepG2 cells were used for heatmap generation (Fig. 1).

Hub genes and their enrichment analysis. Hub genes are highly interconnected genes that might be involved in important cancer-related biological processes and functions. Fig. 2 shows the protein-protein interaction network of the ten hub genes identified by Cytohubba's MCC tool.

When these hub genes were subjected to STRING and enrichment analysis to identify significant GO terms and
Table II. Upregulated and downregulated genes in SIN-treated HepG2 cells compared with SIN-untreated HepG2 cells.

\begin{tabular}{ll}
\hline A, Upregulated genes & \\
\hline No. & Genes \\
\hline 1 & REEP6 \\
2 & CYP1A1 \\
3 & RASGRP2 \\
4 & UGT1A1 \\
\hline
\end{tabular}

\section{B, Downregulated genes}

\begin{aligned} & \hline No. Genes \\ & \hline 1 PLA2G2A \\ & 2 C4BPA \\ & 3 CCL20 \\ & 4 AGXT \\ & 5 BHLHE40 \\ & 6 ITIH3 \\ & 7 CP \\ & 8 FGB \\ & 9 PDE4D \\ & 10 ADGRV1 \\ & 11 IER3 \\ & 12 PGC \\ & 13 SGK1 \\ & 14 IGFBP1 \\ & 15 SERPINE1 \\ & 16 RELN \\ & 17 GK \\ & 18 SERPINA7 \\ & 19 SLC6A14 \\ & 20 MBNL3 \\ & 21 PLIN2 \\ & 22 ORM1 \\ & 23 ORM2 \\ & 24 C5 \\ & 25 IGF2 \\ & 26 SAA4 \\ & 27 SERPINH1 \\ & 28 DKK1 \\ & 29 LOXL4 \\ & 30 CHST15 \\ & 31 INHBE \\ & 32 NAV3 \\ & 34 NR1H4 \\ & 35 DRAM1 \\ & 36 FAM181A \\ & 37 THBS1 \\ & 39 ALDH1A2 \\ & \hline ADGRG1 \\ & \hline GDF15 \\ & \hline\end{aligned}

SIN, sinensetin. 

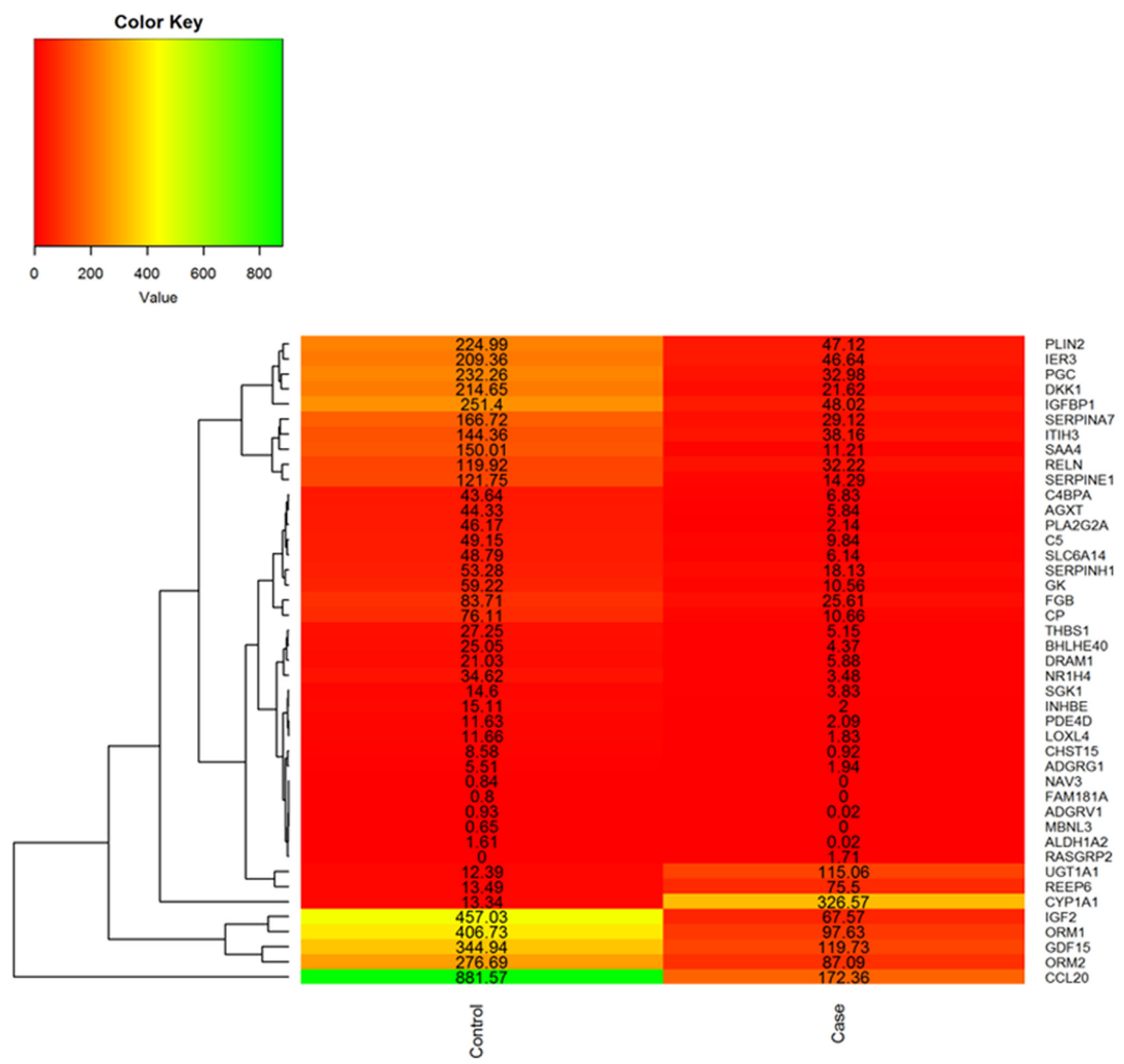

Figure 1. Heatmap of differentially expressed genes. Each row represents a gene and each column represents a control (SIN-untreated HepG2 cells) or case (SIN-treated HepG2 cells) sample. Numbers indicate fragments per kilobase per million mapped reads values. The analysis revealed 43 differentially expressed genes (4 upregulated and 39 downregulated). SIN, sinensetin.

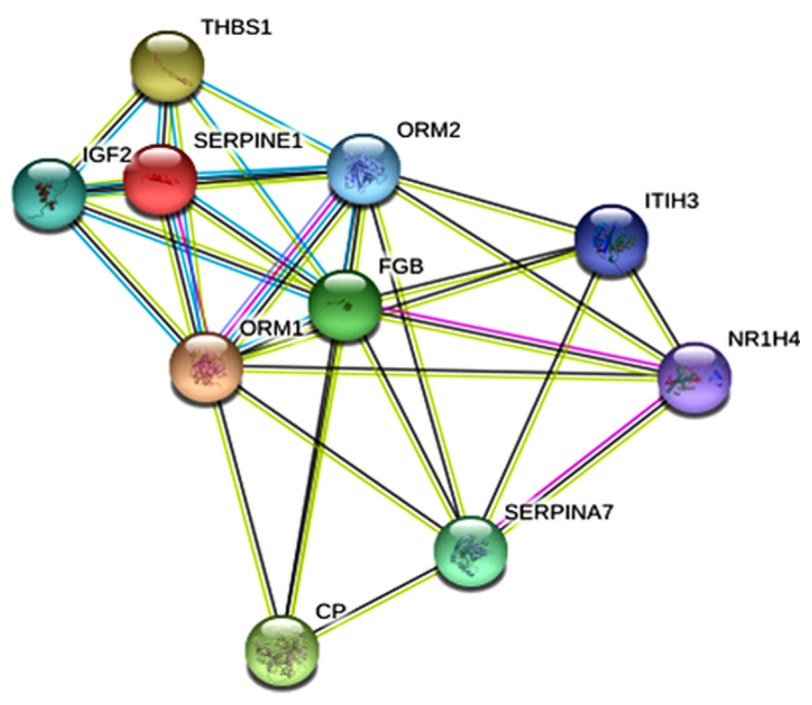

Figure 2. Protein-protein interaction network of hub genes from STRING. Interactions from curated databases, experimentally measured, text-mining and co-expression are shown in blue, pink, green and black, respectively.

KEGG pathways, a list of 101 biological process GO terms and three significant pathways were retrieved. For the ease of analysis and visualization, we did a REVIGO analysis for top ten biological process GO terms showing the lowest FDR values (23). REVIGO indicated that these genes were mainly involved in immune-system responses, regulation of tumor necrosis factor production, regulation of the apoptotic process, regulation of protein metabolism, and transport and secretion processes as shown in the REVIGO scatter plot (Fig. 3). Additionally, KEGG pathway analysis revealed that SERPINE1 and THBS1 modified wild-type human p53 (TP53), complement, coagulation cascade, and proteoglycans in cancer pathways.

Roles of expression of hub genes in cancer. All our hub genes were downregulated. Therefore, we did a comprehensive literature search to figure out roles of these upregulated hub genes in cancer.

Insulin-like growth factor-2 (IGF2). IGF2 is a 67-amino-acid mitogenic peptide hormone involved in the regulation of cell growth, differentiation, and metabolism. It is mainly expressed by the liver, although it can be expressed in many other tissues (24). IGF2 is overexpressed in a variety of cancers. Cancer cells that overexpress IGF2 have a strong tendency to metastasize $(25,26)$. One study has shown that there is a five-fold increase in the expression of IGF2 in HepG2 cells (27). The use of antisense oligodeoxynucleotides (ATON) to halt the translation of IGF2 mRNA has shown 


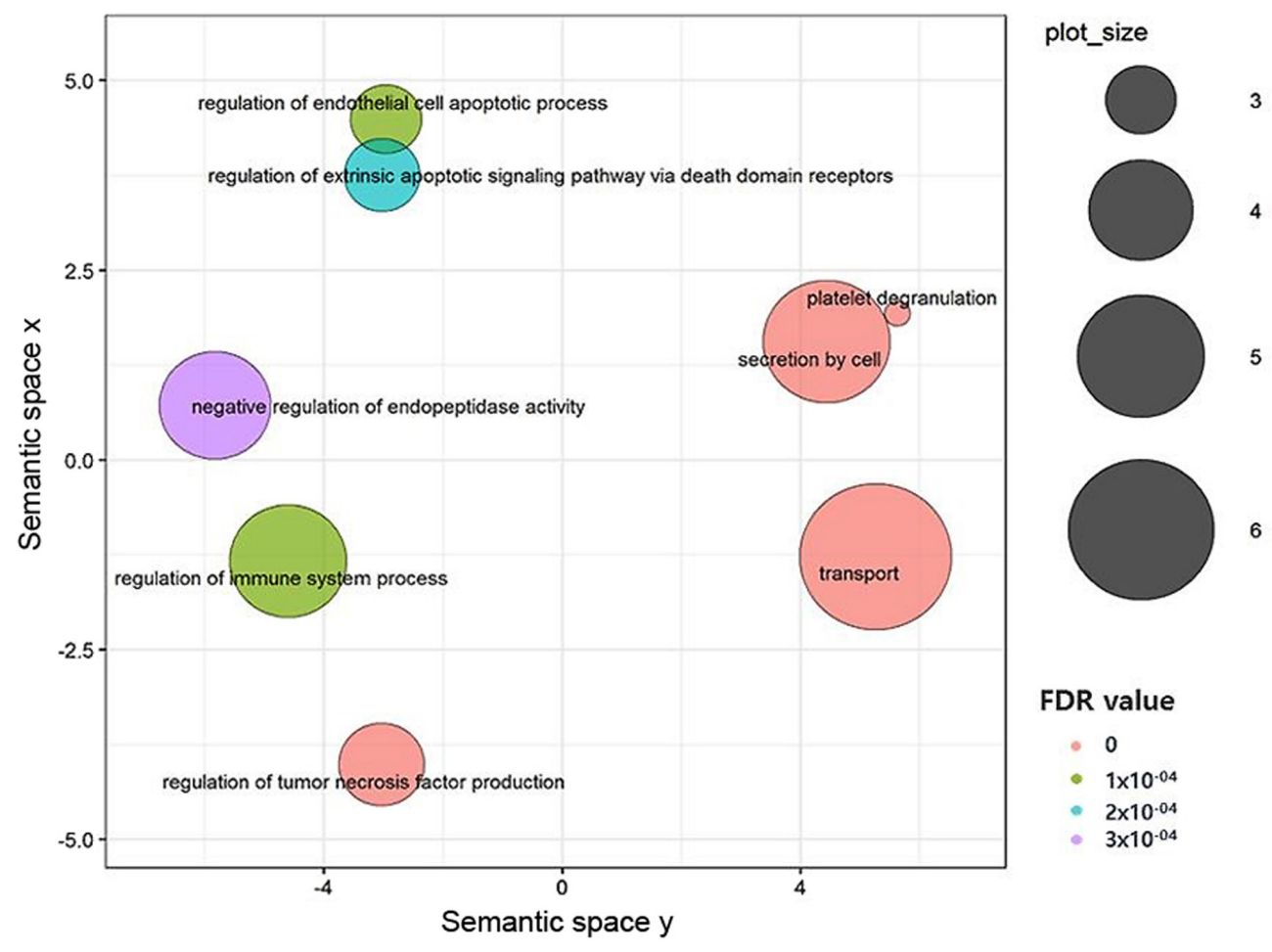

Figure 3. REVIGO scatter plot showing top 10 biological process GO terms with least FDR values. Color indicates the FDR value. Size indicates the frequency of the GO terms in the underlying GO annotation database. GO, Gene Ontology; FDR, false discovery rate.

that a decrease of IGF2 can inhibit the growth of HepG2 cells. Another study has also observed an overexpression of IGF2 in hepatocellular carcinoma cells. Similarly, there is a notable decrease in tumor growth and an increase in mice survival when antibodies against IGF1 and IGF2 are administered (28).

SERPINE1 and SERPINA7. SERPINE1 and SERPINA7 belong to the human SERPIN gene family, which gets its name from its originally identified function of serine proteinase inhibition. However, many of its members also act as chaperones involved in storage, transport, and other roles (29-31). SERPINE1 encodes for a serine proteinase inhibitor. It can inhibit tissue plasminogen activator (tPA) and urokinase (uPA). High levels of SERPINE1 have been associated with low prognosis rate and survival of lung, breast, oral, stomach, and ovarian carcinoma patients (32-34). In addition, reducing the level of SERPINE1 can decrease cell migration in thyroid cancer (35). In relation to HCC, higher levels of SERPINE1 and increased SERPINE1 proteins associated with invasiveness, metastasis, and prognosis in patients with liver cancer have been observed $(36,37)$. SERPINA7 encodes thyroxine-binding globulin (TBG), a human thyroid hormone protein. SERPINA7 has been found to be upregulated in colorectal cancer patients (38). One study on 30 patients with primary liver cancer has found that 22 of them have higher TBG concentrations. Additionally, in 28 out of 31 patients with liver metastasis, TBG concentration is higher than normal (39).

Fibrinogen beta chain (FGB). Fibrinogen is a glycopeptide synthesized by hepatocytes. It is formed by connection of two trimers with each trimer containing a fibrinogen alpha chain that is encoded by the FGA gene, along with the fibrinogen beta chain or gamma chains encoded by FGB or FGG gene, respectively. Increased fibrinogen activity can affect tumor cell growth, progression, and metastasis (40). Moreover, colorectal cancer growth is reduced in fibrinogen-deficient mice (41). The FGB gene is also upregulated in lung carcinomas and breast cancer $(42,43)$. Although we could not find a direct link between upregulation of FGB and HCC, in vitro studies have shown that FGG (another gene involved in fibrinogen formation) upregulation can promote the migration and invasion of HCC whereas knockdown of FGG can significantly inhibit phenotypes (44).

Orosomucoid 1 (ORM1) and orosomucoid 2 (ORM2). The orosomucoid gene family contains two polymorphic genes (ORM1 and ORM2) primarily secreted by the liver, although they are also abundant in the plasma. They encode for acute-phase proteins that are expressed during stressful conditions such as tissue injury, infections, and inflammations (45). It has been reported that ORM genes are over-expressed in breast cancer, bladder cancer, lung cancer, cholangiocarcinoma (bile duct cancer), colorectal cancer, and HCC (46-50). However, the mechanism of how orosomucoid genes affect cancer cells remains unclear. Of particular interest, knockdown of ORM1 can result in decreased proliferation of HCC cells (46).

Nuclear receptor subfamily 1 group H member 4 (NR1H4). NR1H4, also known as farnesoid X receptor (FXR), is mainly expressed in the liver, kidney, intestine, and adrenal gland. It is a member of the nuclear receptor superfamily. It is activated upon binding to bile acid for regulating bile acid homeostasis (51-53). In vitro studies have revealed that the expression of FXR is significantly increased in non-small-cell lung carcinoma (NSCLC), resulting in cell proliferation. Knockdown of NR1H4 can inhibit cell 


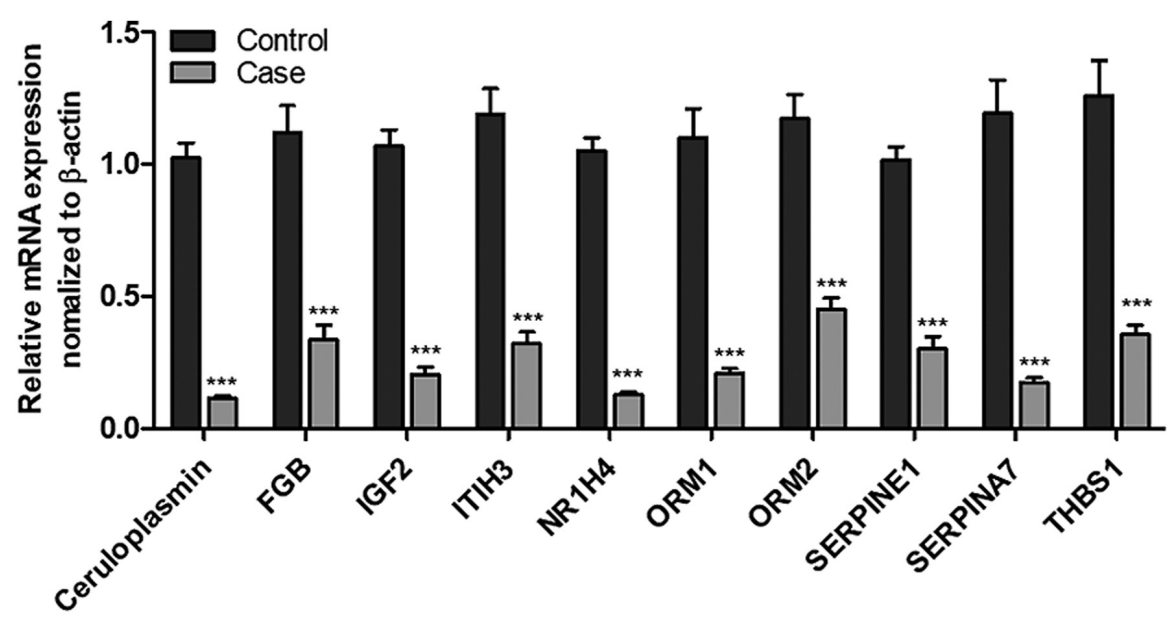

Figure 4. Quantification of genes using RT-qPCR. Hub genes were quantified by RT-qPCR analysis. The expression levels of each gene were suppressed when treated with SIN compared with those in the control group. ${ }^{* * *} \mathrm{P}<0.001$ vs. control. RT-qPCR, reverse transcription-quantitative PCR; FGB, fibrinogen $\beta$ chain; IGF2, insulin-like growth factor 2; ITIH3, inter- $\alpha$-trypsin inhibitor heavy chain 3; NRIH4, nuclear receptor subfamily 1 group H member 4; ORM1/2, orosomucoid 1/2; SERPINE1, serpin family E member 1; SERPINA7, serpin family A member 7; THBS1, thrombospondin 1.

proliferation and xenograft growth in nude mice models A delay in the G1/S transition of cell cycle has also been reported after knockdown of NR1H4 in NSCLC (54). The expression of FXR in esophageal carcinoma is shown to be highly associated with increased tumor size and lymph-node metastasis, whereas deletion of this gene can suppress tumor-cell growth in both in vitro and in vivo studies (55). Some evidence has highlighted the role of FXR in liver carcinogenesis. How FXR promotes cell proliferation has been elucidated in a HepG2 cell line among others by suppressing the expression of p16/INK4a. Downregulation of FXR also shows proliferation-inhibitory effects (56).

Thrombospondin 1 (THBS1). THBS1, a member of the thrombospondins family of proteins, is an important component of the extracellular matrix (57). Upregulation of THBS1 can increase the invasion and migration of gastric cancer cells, prostate cancer, gliomas, pancreatic cancer, and ovarian cancer (58-62). High expression level of THBS1 is associated with invasiveness and progression of hepatocellular carcinoma cells as well as poor prognosis (63). THBS1 expression is also observed in stromal cells surrounding the cancer (64). A study by Lee et al has highlighted the role of THBS1 in HCC tumor progression because suppression of THBS1 can mediate CD47 signaling and decrease the growth of cancerous liver cells (65).

Ceruloplasmin $(C P)$. CP is an enzyme involved in ferroxidase activity, copper transport, amine oxidase activity, and superoxide dismutase activity (66). In breast cancer, elevated $\mathrm{CP}$ levels are associated with metastasis and higher chances of recurrences (67). CP levels are also increased in patients with pancreatic ductal adenocarcinoma, ovarian cancer, bile duct cancer, and cervical cancer (68-71).

Inter-alpha-trypsin inhibitor heavy chain 3 (ITIH3). Inter-alpha-trypsin inhibitors are a family of serine protease inhibitors that are formed by the combination of one light chain and one or two heavy chain proteins. Structurally, two or more heavy chains are covalently linked to hyaluronic acid (HA) which forms the major portion of the cellular matrix (72). Upregulation of ITIH3 expression has been observed in lung cancer and gastric cancer (73-75). The present transcriptome data showed that ITIH3 was downregulated in SIN-treated HepG2 cells than in untreated HepG2 cells. Literature evidence accentuates the role of these hub genes in cancer development when they are expressed at higher than normal levels.

qRT-PCR confirms downregulation of hub genes. Differently expressed, highly integrated hub genes (CP, FGB, IGF2, ITIH3, NRIH4, ORM1, ORM2, SERPINE1, SERPINA7 and THBS1) were quantified for mRNA expression by Qrt-PCR. In agreement with transcriptome analysis data, qRT-PCR analysis results validated the downregulation of these hub genes in SIN-treated cells than in untreated HepG2 cells (Fig. 4). These data further confirm the role of SIN in regulating the expression of hub genes in HepG2 cells.

\section{Discussion}

A transcriptome of an organism describes a small proportion of its genetic codes that can be transcribed into RNA molecules. Post-transcriptional processing of RNA plays a crucial role in terms of producing more variant forms of mRNA (76). Thus, it is clear that studying the whole transcriptome and understanding their modifications can provide an extensive knowledge for developing novel strategies to control diseases. Transcriptome profiling has gained extensive attention in cancer research because it enables disease condition analysis and treatment outcomes. The analysis of RNA-seq data to obtain gene expression and transcriptional changes in cancer supports moral outcomes and treatment options (77). Genes and signaling pathways involved in cancer and treatment outcomes have been identified by microarray and RNA-seq approaches. With the advent of second- and third-generation sequencing technologies, RNA-seq is a significant method owing to its low false-positive results and increased reproducibility rate compared to microarrays (78). It also gives an accurate expression change of transcripts and their isoforms that can help us discover novel transcripts. Transcriptome profiling via RNA-seq has discovered many genes that are potentially involved in the anti-cancer effect of natural compounds like flavonoids. A recent report on 
integrated whole-transcriptome profiling of genes in HCT-116 cancer cells by quercetin treatment has revealed pathways that can regulate cancer progression (79).

Sinensetin (SIN), a polymethoxyflavone present in the citrus family, can inhibit several cancers by regulating oxidative stress of cells (80). Our previous study has displayed an autophagy-mediated anti-cancer potential of SIN in liver cancer cells. In the current research, we performed RNA-seq analysis for SIN-treated and SIN-untreated liver cancer cells (HepG2) to identify critical genes associated with the anti-cancer potential of SIN.

In vitro and in silico techniques have been widely used to study anti-cancer effects of natural compounds and their mechanisms of action. In vivo animal models have also demonstrated different mechanism involved in their abilities to prevent diseases such as cancer, neurodegenerative diseases, and cardiovascular disorders (81). Such studies can help us understand effects of chemotherapeutics on different enzymes, cell signaling protein cascades, and gene expression.

In the current study, a total of 43 differentially expressed genes were identified between SIN-treated and untreated samples in HepG2 cells. Interestingly, most (39/43) of these genes were downregulated while only four were upregulated by treatment with SIN. With the help of STRING and Cytohubba, we identified ten hub genes from the DEG list. Enrichment analysis indicated that these hub genes were mainly involved in immune-system responses and regulation of tumor necrosis factor production, apoptosis, and protein metabolism. As presented in detail in the results section, we did an extensive literature survey on these identified hub genes, highlighting their roles in tumor growth, tumor invasiveness, poor prognosis, and recurrence in various cancers upon upregulation of their expression. RNA-seq analysis of HepG 2 cells treated with SIN showed downregulation of these hub genes. Literature analysis sheds light on how downregulation of these hub genes might mediate anti-cancer processes. qRT-PCR data confirmed that expression levels of these hub genes were consistent with RNA-seq data. Hub genes CP, FGB, IGF2, ITIH3, NRIH4, ORM1, ORM2, SERPINE1, SERPINA7 and THBS1 are highly expressed in several cancers, including liver, lung, pancreatic, and cervical cancers. Significant downregulation of these genes upon SIN treatment showed its prominent capacity in suppressing these genes in HepG2 cells. The confirmation of expression data revealed that these genes could emerge as attractive therapeutic targets in the treatment of liver cancer. Furthermore, RNA-seq and relative expression data strengthened the argument that SIN is a strong anti-cancer agent in HCC.

In conclusion, in the current study, transcriptome analysis of SIN-treated HepG2 cells by next generation sequencing supported its anti-cancer effect. Analysis of DEGs provided a strong insight on the involvement of hub genes related to cancer progression. Results of this study indicate that SIN might induce HCC cell death by regulating the expression of these genes. The objective of this study was to identify the related gene on SIN anti-cancer effect using transcriptome analysis. This paper highlights the necessity for further studies to support anti-cancer effect of those genes.

\section{Acknowledgements}

Not applicable.

\section{Funding}

The present study was supported by grants from the National Research Foundation funded by the Ministry of Science and ICT, Republic of Korea (grant nos. 2012M3A9B8019303 and 2020R1A2B5B01001807).

\section{Availability of data and materials}

The datasets used and/or analyzed during the current study are available from the corresponding author on reasonable request.

\section{Authors' contributions}

GSK and KWL conceived, designed and performed the experiments. SMK, SR and AMK organized focus group discussion, collected and analyzed all study data, and prepared the final manuscript. SMK, PV, SEH and HHK performed some experiments and revised the final manuscript. GSK and SMK confirm the authenticity of all the raw data. All authors read and approved the final manuscript.

\section{Ethics approval and consent to participate}

Not applicable.

\section{Patient consent for publication}

Not applicable.

\section{Competing interests}

The authors declare that they have no competing interests.

\section{References}

1. Li J, Fu Y, Hu X and Xiong Y: Psoralidin inhibits the proliferation of human liver cancer cells by triggering cell cycle arrest, apoptosis and autophagy and inhibits tumor growth in vivo. J BUON 24: 1950-1955, 2019.

2. Sakin A, Sahin S, Atci MM, Yasar N, Geredeli C, Aribal S, Alemdar A, Karatas F and Cihan S: Factors affecting survival in patients with isolated liver-metastatic colorectal cancer treated with local ablative or surgical treatments for liver metastasis. J BUON 24: 1801-1808, 2019.

3. Zhang X, Lv J, Luo H, Liu Z, Xu C, Zhou D, Tang L, Zhang Z, Liu J, Xiao M, et al: Nucleostemin promotes hepatocellular carcinoma by regulating the function of STAT3. Exp Cell Res 387: 111748, 2020.

4. Xu L, Wang R, Ziegelbauer J, Wu WW, Shen RF, Juhl H, Zhang Y, Pelosof L and Rosenberg AS: Transcriptome analysis of human colorectal cancer biopsies reveals extensive expression correlations among genes related to cell proliferation, lipid metabolism, immune response and collagen catabolism. Oncotarget 8: 74703-74719, 2017.

5. Xu Y, Lv X, Yang G, Zhan J, Li M, Long T, Ho CT and Li S: Simultaneous separation of six pure polymethoxyflavones from sweet orange peel extract by high performance counter current chromatography. Food Chem 292: 160-165, 2019.

6. Kang SI, Shin HS and Kim SJ: Sinensetin enhances adipogenesis and lipolysis by increasing cyclic adenosine monophosphate levels in 3T3-L1 adipocytes. Biol Pharm Bull 38: 552-558, 2015.

7. Yu T, Zhang H and Qi H: Transcriptome profiling analysis reveals biomarkers in colon cancer samples of various differentiation. Oncol Lett 16: 48-54, 2018. 
8. Luo J, Dai X, Hu H, Chen J, Zhao L, Yang C, Sun J, Zhang L, Wang Q, Xu S, et al: Fluzoparib increases radiation sensitivity of non-small cell lung cancer (NSCLC) cells without BRCA1/2 mutation, a novel PARP1 inhibitor undergoing clinical trials. J Cancer Res Clin Oncol 146: 721-737, 2020.

9. Lin KH, Huang MY, Cheng WC, Wang SC, Fang SH, Tu HP, Su CC, Hung YL, Liu PL, Chen CS, et al: RNA-seq transcriptome analysis of breast cancer cell lines under shikonin treatment. Sci Rep 8: 2672, 2018

10. Wang D, Zhao L, Wang D, Liu J, Yu X, Wei Y and Ouyang Z: Transcriptome analysis and identification of key genes involved in 1-deoxynojirimycin biosynthesis of mulberry (Morus alba L.). PeerJ 6: e5443, 2018.

11. Zhang Y, Kang Z, Lv D, Zhang X, Liao Y, Li Y, Liu R, Li P, Tong M, Tian J, et al: Longitudinal whole-genome sequencing reveals the evolution of MPAL. Cancer Genet 240: 59-65, 2020.

12. Niemira M, Collin F, Szalkowska A, Bielska A, Chwialkowska K, Reszec J, Niklinski J, Kwasniewski M and Kretowski A: Molecular signature of subtypes of non-small-cell lung cancer by large-scale transcriptional profiling: Identification of key modules and genes by Weighted Gene Co-Expression Network Analysis (WGCNA). Cancers (Basel) 12: E37, 2019.

13. Wang Z, Zhang Z, Zhang $\mathrm{C}$ and $\mathrm{Xu}$ Y: Identification of potential pathogenic biomarkers in clear cell renal cell carcinoma. Oncol Lett 15: 8491-8499, 2018.

14. Stark R, Grzelak M and Hadfield J: RNA sequencing: The teenage years. Nat Rev Genet 20: 631-656, 2019.

15. Liu Y, Gu HY, Zhu J, Niu YM, Zhang C and Guo GL: Identification of hub genes and key pathways associated with bipolar disorder based on weighted gene co-expression Network Analysis. Front Physiol 10: 1081, 2019.

16. Trapnell C, Pachter L and Salzberg SL: TopHat: Discovering splice junctions with RNA-Seq. Bioinformatics 25: 1105-1111, 2009.

17. Trapnell C, Williams BA, Pertea G, Mortazavi A, Kwan G, van Baren MJ, Salzberg SL, Wold BJ and Pachter L: Transcript assembly and quantification by RNA-Seq reveals unannotated transcripts and isoform switching during cell differentiation. Nat Biotechnol 28: 511-515, 2010.

18. Benjamini Y, Drai D, Elmer G, Kafkafi N and Golani I: Controlling the false discovery rate in behavior genetics research. Behav Brain Res 125: 279-284, 2001.

19. Szklarczyk D, Gable AL, Lyon D, Junge A, Wyder S, Huerta-Cepas J, Simonovic M, Doncheva NT, Morris JH, Bork P, et al: STRING v11: Protein-protein association networks with increased coverage, supporting functional discovery in genome-wide experimental datasets. Nucleic Acids Res 47: D607-D613, 2019.

20. Shannon P, Markiel A, Ozier O, Baliga NS, Wang JT, Ramage D, Amin N, Schwikowski B and Ideker T: Cytoscape: A software environment for integrated models of biomolecular interaction networks. Genome Res 13: 2498-2504, 2003

21. Supek F, Bošnjak M, Škunca N and Šmuc T: REVIGO summarizes and visualizes long lists of gene ontology terms. PLoS One 6: e21800, 2011.

22. Kim SM,Ha SE,Ho JL, Rampogu S, Vetrivel P, Kim HH, Saralamma VVG, Lee KW and Kim GS: Sinensetin Induces Autophagic Cell Death through p53-Related AMPK/mTOR Signaling in Hepatocellular Carcinoma HepG2 Cells. Nutrients 12: 2462, 2020

23. Xiong H, Guo H, Xie Y, Zhao L, Gu J, Zhao S, Li J and Liu L: RNAseq analysis reveals pathways and candidate genes associated with salinity tolerance in a spaceflight-induced wheat mutant. Sci Rep 7: 2731, 2017.

24. Livingstone C: IGF2 and cancer. Endocr Relat Cancer 20 R321-R339, 2013.

25. Macaulay VM: Insulin-like growth factors and cancer. Br J Cancer 65: 311-320, 1992.

26. Guerra FK, Eijan AM, Puricelli L, Alonso DF, Bal de Kier Joffé E, Kornblihgtt AR, Charreau EH and Elizalde PV: Varying patterns of expression of insulin-like growth factors I and II and their receptors in murine mammary adenocarcinomas of different metastasizing ability. Int J Cancer 65: 812-820, 1996

27. Lin SB, Hsieh SH, Hsu HL, Lai MY, Kan LS and Au LC: Antisense oligodeoxynucleotides of IGF-II selectively inhibit growth of human hepatoma cells overproducing IGF-II. J Biochem 122: 717-722, 1997.

28. Martinez-Quetglas I, Pinyol R, Dauch D, Torrecilla S, Tovar V, Moeini A, Alsinet C, Portela A, Rodriguez-Carunchio L, Solé M, et al: IGF2 Is up-regulated by epigenetic mechanisms in hepatocellular carcinomas and is an actionable oncogene product in experimental models. Gastroenterology 151: 1192-1205, 2016.
29. Andreasen PA, Kjøller L, Christensen L and Duffy MJ: The urokinase-type plasminogen activator system in cancer metastasis: A review. Int J Cancer 72: 1-22, 1997.

30. Hundsdorfer B, Zeilhofer HF, Bock KP, Dettmar P, Schmitt M and Horch HH: The prognostic importance of urinase type plasminogen activators (uPA) and plasminogen activator inhibitors (PAI-1) in the primary resection of oral squamous cell carcinoma. Mund Kiefer Gesichtschir 8: 173-179, 2004 (In German).

31. Annecke K, Schmitt M, Euler U, Zerm M, Paepke D, Paepke S, von Minckwitz G, Thomssen C and Harbeck N: uPA and PAI-1 in breast cancer: Review of their clinical utility and current validation in the prospective NNBC-3 trial. Adv Clin Chem 45: 31-45, 2008.

32. Harbeck N, Schmitt M, Paepke S, Allgayer H and Kates RE: Tumor-associated proteolytic factors UPA and PAI-1: Critical appraisal of their clinical relevance in breast cancer and their integration into decision-support algorithms. Crit Rev Clin Lab Sci 44: 179-201, 2007.

33. Vairaktaris E, Yapijakis C, Psyrri A, Spyridonidou S, Yannopoulos A, Lazaris A, Vassiliou S, Ferekidis E, Vylliotis A, Nkenke E, et al: Loss of tumour suppressor p16 expression in initial stages of oral oncogenesis. Anticancer Res 27: 979-984, 2007.

34. Li L, Zhu Z, Zhao Y, Zhang Q, Wu X, Miao B, Cao J and Fei S: FN1, SPARC, and SERPINE1 are highly expressed and significantly related to a poor prognosis of gastric adenocarcinoma revealed by microarray and bioinformatics. Sci Rep 9: 7827, 2019.

35. Yu XM, Jaskula-Sztul R, Georgen MR, Aburjania Z, Somnay YR, Leverson G, Sippel RS, Lloyd RV, Johnson BP and Chen H: Notch1 signaling regulates the aggressiveness of differentiated thyroid cancer and inhibits SERPINE1 expression. Clin Cancer Res 22: 3582-3592, 2016

36. Zheng Q, Tang ZY, Xue Q, Shi DR, Song HY and Tang HB: Invasion and metastasis of hepatocellular carcinoma in relation to urokinase-type plasminogen activator, its receptor and inhibitor. J Cancer Res Clin Oncol 126: 641-646, 2000.

37. Divella R, Mazzocca A, Gadaleta C, Simone G, Paradiso A, Quaranta $\mathrm{M}$ and Daniele A: Influence of plasminogen activator inhibitor-1 (SERPINE1) 4G/5G polymorphism on circulating SERPINE-1 antigen expression in HCC associated with viral infection. Cancer Genomics Proteomics 9: 193-198, 2012.

38. Holm M, Saraswat M, Joenväärä S, Ristimäki A, Haglund C and Renkonen R: Colorectal cancer patients with different C-reactive protein levels and 5-year survival times can be differentiated with quantitative serum proteomics. PLoS One 13: e0195354, 2018.

39. Berger HR, Creech MK, Hannoush Z, Watanabe Y, Kargi A and Weiss RE: A novel mutation causing complete thyroid binding globulin deficiency (Tbg-Cd Mia) in a male with coexisting graves disease. AACE Clin Case Rep 3: e134-e139, 2017.

40. Falanga A, Marchetti M and Vignoli A: Coagulation and cancer: Biological and clinical aspects. J Thromb Haemost 11:223-233, 2013

41. Adams GN, Rosenfeldt L, Frederick M, Miller W, Waltz D, Kombrinck K, McElhinney KE, Flick MJ, Monia BP, Revenko AS, et al: Colon cancer growth and dissemination relies upon thrombin, stromal PAR-1, and fibrinogen. Cancer Res 75: 4235-4243, 2015.

42. Ciereszko A, Dietrich MA, Słowińska M, Nynca J, Ciborowski M, Kisluk J, Michalska-Falkowska A, Reszec J, Sierko E and Nikliński J: Identification of protein changes in the blood plasma of lung cancer patients subjected to chemotherapy using a 2D-DIGE approach. PLoS One 14: e0223840, 2019.

43. Dowling P, Palmerini V, Henry M, Meleady P, Lynch V, Ballot J, Gullo G, Crown J, Moriarty M and Clynes M: Transferrin-bound proteins as potential biomarkers for advanced breast cancer patients. BBA Clin 2: 24-30, 2014.

44. Zhang X, Wang F, Huang Y, Ke K, Zhao B, Chen L, Liao N, Wang L, Li Q, Liu X, et al: FGG promotes migration and invasion in hepatocellular carcinoma cells through activating epithelial to mesenchymal transition. Cancer Manag Res 11: 1653-1665, 2019.

45. Lee YS, Choi JW, Hwang I, Lee JW, Lee JH, Kim AY, Huh JY, Koh YJ, Koh GY, Son HJ, et al: Adipocytokine orosomucoid integrates inflammatory and metabolic signals to preserve energy homeostasis by resolving immoderate inflammation. J Biol Chem 285: 22174-22185, 2010.

46. Li F, Yu Z, Chen P, Lin G, Li T, Hou L, Du Y and Tan W: The increased excretion of urinary orosomucoid 1 as a useful biomarker for bladder cancer. Am J Cancer Res 6: 331-340, 2016.

47. Ayyub A, Saleem M, Fatima I, Tariq A, Hashmi N and Musharraf SG: Glycosylated alpha-1-acid glycoprotein 1 as a potential lung cancer serum biomarker. Int J Biochem Cell Biol 70: 68-75, 2016 
48. Rucksaken R, Charoensuk L, Pinlaor P, Pairojkul C, Khuntikeo N and Pinlaor S: Plasma orosomucoid 2 as a potential risk marker of cholangiocarcinoma. Cancer Biomark 18: 27-34, 2017.

49. Falleti E, Pirisi M, Fabris C, Bortolotti N, Soardo G, Toniutto P, Gonano F and Bartoli E: Increase of serum alpha 1-acid glycoprotein despite the decline of liver synthetic function in cirrhotics with hepatocellular carcinoma. Eur J Clin Chem Clin Biochem 31: 407-411, 1993.

50. Alexander H, Stegner AL, Wagner-Mann C, Du Bois GC, Alexander $\mathrm{S}$ and Sauter ER: Proteomic analysis to identify breast cancer biomarkers in nipple aspirate fluid. Clin Cancer Res 10: 7500-7510, 2004.

51. Forman BM, Goode E, Chen J, Oro AE, Bradley DJ, Perlmann T, Noonan DJ, Burka LT, McMorris T, Lamph WW, et al: Identification of a nuclear receptor that is activated by farnesol metabolites. Cell 81: 687-693, 1995.

52. Sinal CJ, Tohkin M, Miyata M, Ward JM, Lambert G and Gonzalez FJ: Targeted disruption of the nuclear receptor FXR/BAR impairs bile acid and lipid homeostasis. Cell 102: 731-744, 2000.

53. Lu TT, Makishima M, Repa JJ, Schoonjans K, Kerr TA, Auwerx J and Mangelsdorf DJ: Molecular basis for feedback regulation of bile acid synthesis by nuclear receptors. Mol Cell 6: 507-515, 2000.

54. You W, Chen B, Liu X, Xue S, Qin H and Jiang H: Farnesoid X receptor, a novel proto-oncogene in non-small cell lung cancer, promotes tumor growth via directly transactivating CCND1. Sci Rep 7: 591, 2017

55. Guan B, Li H, Yang Z, Hoque A and Xu X: Inhibition of farnesoid $X$ receptor controls esophageal cancer cell growth in vitro and in nude mouse xenografts. Cancer 119: 1321-1329, 2013.

56. Guo F, Xu Z, Zhang Y, Jiang P, Huang G, Chen S, Lyu X, Zheng P, Zhao X, Zeng Y, et al: FXR induces SOCS3 and suppresses hepatocellular carcinoma. Oncotarget 6: 34606-34616, 2015

57. Adams JC and Lawler J: The thrombospondins. Int J Biochem Cell Biol 36: 961-968, 2004

58. Huang T, Wang L, Liu D, Li P, Xiong H, Zhuang L, Sun L, Yuan X and Qiu H: FGF7/FGFR2 signal promotes invasion and migration in human gastric cancer through upregulation of thrombospondin-1. Int J Oncol 50: 1501-1512, 2017.

59. Firlej V, Mathieu JR, Gilbert C, Lemonnier L, Nakhlé J, Gallou-Kabani C, Guarmit B, Morin A, Prevarskaya N, Delongchamps NB, et al: Thrombospondin-1 triggers cell migration and development of advanced prostate tumors. Cancer Res 71: 7649-7658, 2011.

60. Perez-Janices N, Blanco-Luquin I, Tuñón MT, Barba-Ramos E, Ibáñez B, Zazpe-Cenoz I, Martinez-Aguillo M, Hernandez B Martínez-Lopez E, Fernández AF, et al: EPB41L3, TSP-1 and RASSF2 as new clinically relevant prognostic biomarkers in diffuse gliomas. Oncotarget 6: 368-380, 2015.

61. Nie S, Lo A, Wu J, Zhu J, Tan Z, Simeone DM, Anderson MA, Shedden KA, Ruffin MT and Lubman DM: Glycoprotein biomarker panel for pancreatic cancer discovered by quantitative proteomics analysis. J Proteome Res 13: 1873-1884, 2014.

62. Lyu T, Jia N, Wang J, Yan X, Yu Y, Lu Z, Bast RC Jr, Hua K and Feng W: Expression and epigenetic regulation of angiogenesis-related factors during dormancy and recurrent growth of ovarian carcinoma. Epigenetics 8: 1330-1346, 2013.

63. Poon RT, Chung KK, Cheung ST, Lau CP, Tong SW, Leung KL, Yu WC, Tuszynski GP and Fan ST: Clinical significance of thrombospondin 1 expression in hepatocellular carcinoma. Clin Cancer Res 10: 4150-4157, 2004.

64. Hayashi K, Kurohiji T and Shirouzu K: Localization of thrombospondin in hepatocellular carcinoma. Hepatology 25: 569-574, 1997.

65. Lee TK, Cheung VC, Lu P, Lau EY, Ma S, Tang KH, Tong M, Lo $\mathrm{J}$ and $\mathrm{Ng}$ IO: Blockade of CD47-mediated cathepsin S/protease-activated receptor 2 signaling provides a therapeutic target for hepatocellular carcinoma. Hepatology 60: 179-191, 2014.
66. Tórsdóttir G, Gudmundsson G, Kristinsson J, Snaedal J and Jóhannesson T: Ceruloplasmin and superoxide dismutase (SOD1) in heterozygotes for Wilson disease: A case control study. Neuropsychiatr Dis Treat 5: 55-59, 2009.

67. Schapira DV and Schapira M: Use of ceruloplasmin levels to monitor response to therapy and predict recurrence of breast cancer. Breast Cancer Res Treat 3: 221-224, 1983.

68. Brandi J, Dalla Pozza E, Dando I, Biondani G, Robotti E, Jenkins R, Elliott V, Park K, Marengo E, Costello E, et al: Secretome protein signature of human pancreatic cancer stem-like cells. J Proteomics 136: 1-12, 2016.

69. Han IW, Jang JY, Kwon W, Park T, Kim Y, Lee KB and Kim SW: Ceruloplasmin as a prognostic marker in patients with bile duct cancer. Oncotarget 8: 29028-29037, 2017.

70. Upadhya S, Upadhya S and Prabhu KS: Serum glycoconjugates and ceruloplasmin in cancer of uterine cervix. Indian J Clin Biochem 17: 20-24, 2002.

71. Arumanayagam M, Wong FW, Rogers M and Swaminathan R: Serum ceruloplasmin, plasma copper concentration and copper to ceruloplasmin ratio in cervical carcinoma. Gynecol Obstet Invest 35: 175-178, 1993.

72. Huang L, Yoneda M and Kimata K: A serum-derived hyaluronan-associated protein (SHAP) is the heavy chain of the inter alpha-trypsin inhibitor. J Biol Chem 268: 26725-26730, 1993.

73. Chatterji B and Borlak J: A 2-DE MALDI-TOF study to identify disease regulated serum proteins in lung cancer of c-myc transgenic mice. Proteomics 9: 1044-1056, 2009.

74. Heo SH, Lee SJ, Ryoo HM, Park JY and Cho JY: Identification of putative serum glycoprotein biomarkers for human lung adenocarcinoma by multilectin affinity chromatography and LC-MS/MS. Proteomics 7: 4292-4302, 2007.

75. Chong PK, Lee H, Zhou J, Liu SC, Loh MC, Wang TT, Chan SP, Smoot DT, Ashktorab H, So JB, et al: ITIH3 is a potential biomarker for early detection of gastric cancer. J Proteome Res 9: 3671-3679, 2010.

76. Pertea M: The human transcriptome: An unfinished story. Genes (Basel) 3: 344-360, 2012.

77. Cajigas-Du Ross CK, Martinez SR, Woods-Burnham L, Durán AM, Roy S, Basu A, Ramirez JA, Ortiz-Hernández GL, Ríos-Colón L, Chirshev E, et al: RNA sequencing reveals upregulation of a transcriptomic program associated with stemness in metastatic prostate cancer cells selected for taxane resistance. Oncotarget 9: 30363-30384, 2018.

78. Zhao S, Fung-Leung WP, Bittner A, Ngo K and Liu X: Comparison of RNA-Seq and microarray in transcriptome profiling of activated T cells. PLoS One 9: e78644, 2014.

79. Zhang Z, Li B, Xu P and Yang B: Integrated whole transcriptome profiling and bioinformatics analysis for revealing regulatory pathways associated with quercetin-induced apoptosis in HCT-116 cells. Front Pharmacol 10: 798, 2019.

80. Xiong YJ, Deng ZB, Liu JN, Qiu JJ, Guo L, Feng PP, Sui JR, Chen DP and Guo HS: Enhancement of epithelial cell autophagy induced by sinensetin alleviates epithelial barrier dysfunction in colitis. Pharmacol Res 148: 104461, 2019.

81. Forni C, Facchiano F, Bartoli M, Pieretti S, Facchiano A, D'Arcangelo D, Norelli S, Valle G, Nisini R, Beninati S, et al: Beneficial role of phytochemicals on oxidative stress and age-related diseases. BioMed Res Int 2019: 8748253, 2019.

This work is licensed under a Creative Commons Attribution-NonCommercial-NoDerivatives 4.0 International (CC BY-NC-ND 4.0) License. 